\title{
Automatic nasal continuous positive airway pressure titration in the laboratory: patient
} outcomes

\author{
J R Stradling, C Barbour, D J Pitson, R J O Davies
}

\begin{abstract}
Background - Manual titration of nasal continuous positive airway pressure (NCPAP) treatment for obstructive sleep apnoea (OSA) is time consuming and expensive. There are now "intelligent" NCPAP machines that try to find the ideal pressure for a patient by monitoring a combination of apnoeas, hypopnoeas, inspiratory flow limitation, and snoring. Although these machines usually find similar pressures to skilled technicians, it is not clear if their use in the sleep laboratory influences subsequent acceptance by patients. This study addresses this question.
\end{abstract}

Methods - One hundred and twenty two patients undergoing a trial of NCPAP were randomly allocated to either manual or automatic (Horizon, DeVilbiss) titration of pressure during their first night on NCPAP in a hospital sleep laboratory. The primary outcome (available on 112 patients) was the acceptance of NCPAP or otherwise six weeks following the initial titration night. Baseline indicators of severity were compared between the groups, as were the pressures selected and the subsequent improvement in the sleepiness of the patients.

Results - The initial severity of OSA was not significantly different in the two groups and the mean (SD) NCPAP pressures were similar (manual 8.7 (2.5) $\mathrm{cm} \mathrm{H}_{2} \mathrm{O}$, automatic $\left.8.2(2.1) \mathrm{cm} \mathrm{H}_{2} \mathrm{O}\right)$. The percentage of patients successfully established on CPAP at six weeks was $64 \%$ and $73 \%$ for the manual and automatic groups, respectively; $13 \%$ and $2 \%$, respectively, in the manual and automatic groups had given up completely $(p<0.05)$, and there were about equal numbers $(23 \%$ versus $25 \%$ ) in the two groups who were still undecided.

Conclusions - The substitution of automatic NCPAP titration for manual titration during the first night of NCPAP in patients with OSA does not reduce the number accepting the treatment at six weeks and may slightly improve it. This has important cost saving potential.

(Thorax 1997;52:72-75)

Keywords: obstructive sleep apnoea, nasal continuous positive airway pressure, automatic.
Nasal continuous positive airway pressure (NCPAP) is the most effective therapy for obstructive sleep apnoea (OSA). Patients are usually admitted to hospital overnight to start treatment. During monitored sleep the lowest pressure that is required to abolish the apnoeas and arousals is established by manually increasing and lowering the airway pressure and watching the effects on airflow, snoring, arterial oxygenation, and some measure of sleep fragmentation such as movement or electroencephalographic (EEG) disturbance. This is usually done over part or all of the night so that periods requiring the highest pressures, such as the supine posture and rapid eye movement (REM) sleep, are included in the titration period. However, it is labour intensive, time consuming, and expensive. When it was thought that sleep monitoring with EEG was necessary during NCPAP titration, a technician was required to tend to the equipment anyway. However, in a study that assessed the adequacy of respiratory variables alone (airflow, chest wall movements, oximetry, and snoring) it was established that EEG monitoring during CPAP titration was not required, ${ }^{1}$ so an automatic NCPAP machine capable of finding the optimal pressure using feedback signals such as airflow and snoring could mean a substantial saving in technician time. Such a system might also track the required pressure more assiduously than is usually possible manually, and hence the pressure on which the patient is discharged home might be more accurately prescribed. Such NCPAP machines are now available. They use a variety of signals to assess when to raise or lower their pressure, such as airflow or mask pressure (for apnoeas, hypopnoeas, and flow limitation) and snoring. The pressures found by these machines generally agree well with those established by skilled technicians. ${ }^{2-8}$

As well as theoretical advantages, these automatic NCPAP machines have some theoretical disadvantages. Because they inevitably "hunt" around the ideal pressure and may respond inappropriately on some occasions, they could result in a more disturbed first night on CPAP and reduce the patient's enthusiasm to try the treatment at home. In addition, if there was a difference between the manual pressure and the machine derived pressure (either too low or too high), this might lessen the efficacy of NCPAP and lead to early abandonment by the patient. 
This study was designed to establish if the substitution of automatic for manual NCPAP titration on the patient's first night improved or reduced NCPAP acceptance at five weeks.

\section{Methods \\ PATIENTS \\ All patients who required a trial of NCPAP at the Oxford Sleep Clinic over an eight month period from 16 May 1995 to 23 January 1996 were studied. The criteria for inclusion in the trial of NCPAP were: (1) significant disabling symptoms of OSA (usually excessive sleepiness quantified by the Epworth Sleepiness Scale ${ }^{9}$ ); (2) evidence of both sleep disturbance (multiple movement arousals on video and pulse rate rises) and upper airway obstruction (hypoxic dips, heavy snoring, apnoeas seen on video (Visi Lab System, Stowood Scientific Instruments, Oxford, UK)) on the sleep study; and (3) a willingness to try NCPAP following a detailed explanation of what it entailed.}

\section{NCPAP TITRATION TECHNIQUES Manual titration}

Following a second full explanation of NCPAP on the evening of admission, patients were allowed to fall asleep wearing their nasal mask, with the pressure set to about $3 \mathrm{~cm} \mathrm{H}_{2} \mathrm{O}$. Once the patient was asleep and was experiencing upper airway obstruction the pressure was raised until all evidence of obstruction and its consequences disappeared (absence of snoring, movement arousals, pulse rate rises, and dips in oxygen saturation). The pressure was then reduced until obstructive events returned and then increased again. This cycle was repeated until the correct pressure level was confidently assessed. This process usually took about two hours and thereafter no further titration was performed. The following morning the tracings were reviewed for any return of events. If there had been a return of events, with the mask satisfactorily in place, then the pressure at which the patient was sent home was increased by $1-2 \mathrm{~cm} \mathrm{H}_{2} \mathrm{O}$. This usually occurred if there had been no supine sleep during the supervised first two hours. The pressure was then kept at the same level until the follow up visit six weeks later.

\section{Automatic titration}

Automatic titration was similar in all respects to manual titration except that pressure adjustment was performed automatically by the DeVilbiss Horizon system (model 7354I) throughout the night. This device senses changes in airflow to the mask and monitors snoring. It responds to apnoeas, hypopnoeas, and snoring by raising the pressure which starts at a low of $3 \mathrm{~cm} \mathrm{H}_{2} \mathrm{O}$. The length and size of apnoeas and hypopnoeas to which it will respond can be programmed. The manufacturer's default settings for events are those lasting more than 10 seconds in length; an apnoea is an $85 \%$ fall in flow signal and an hypopnoea is a $60 \%$ fall. We found that the definition of an hypopnoea had to be extended to 40 seconds in order to prevent the device incorrectly raising its pressure in response to the normal non-obstructive hypopnoeas of REM sleep.

The following morning the automatic pressure profile was reviewed on the Visi Lab system. The single pressure then chosen for that patient to use at home was the lowest that abolished most of the obstructive events and was not necessarily the highest pressure ever reached.

PROTOCOL

Two patients per night, on average two nights per week, were randomly assigned to one of two sleep laboratories, one equipped with a manual titration system and the other with the DeVilbiss automatic CPAP equipment. Following the titration night the patients were sent home with fixed pressure CPAP systems (Revitaliser, DeVilbiss) and reviewed in the clinic about six weeks later. During this period they had telephone access to specialist nurses so that any problems could be solved promptly. It was stressed that this period was a trial and that patients were under no obligation to accept an NCPAP system long term. We explained that NCPAP is prescribed for symptoms and that it is up to patients themselves to decide if the benefits outweigh the disadvantages.

At the follow up appointment the patients were classified into one of three categories: (1) those who were successfully established on NCPAP and happy to continue indefinitely (successful); (2) those who were not yet sure whether they wanted to continue but were prepared to try for longer (undecided); and (3) those who had given up and were not prepared to try again (failed).

\section{DATA ANALYSIS}

Unpaired $t$ testing was used to assess whether those assigned either to manual or automatic titration differed in their baseline characteristics. Unpaired $t$ testing was also used both to assess differences in the CPAP pressures chosen by the two techniques and the Epworth Sleepiness Scale (ESS) in the two groups at the six week follow up appointment. The numbers in each category (successful, undecided, failed) for manual and automatic titration were compared by $\chi^{2}$ analysis.

\section{Results}

One hundred and twenty two patients were initially randomised for the study at CPAP booking. However, nine cancelled (four manual, five automatic) and their space in the laboratory could not be filled in time. On those nights the single remaining patient was always titrated in the manual laboratory regardless of randomisation, thus (in error) four patients received manual instead of the intended automatic titration. Hence, there were 61 subjects in the manual group and 52 in the automatic group. One subject in the automatic group 
Table 1 Mean (SD) data for the manual and automatic CPAP titration groups

\begin{tabular}{lcc}
\hline & $\begin{array}{c}\text { Manual } \\
(n=61)\end{array}$ & $\begin{array}{c}\text { Automatic } \\
(n=52)\end{array}$ \\
\hline Baseline data: & & \\
$>4 \% \mathrm{SaO}_{2}$ dips/hour & $20.0(18.6)$ & $16.6(18.4)$ \\
Pulse rises/hour & $34.2(20.0)$ & $31.0(21.5)$ \\
BMI & $33.7(6.21)$ & $32.5(5.4)$ \\
Neck circumference (in) & $17.3(1.7)$ & $17.1(1.6)$ \\
Epworth Sleepiness Scale & $14.4(4.6)$ & $14.7(5.2)$ \\
Titration data: & & \\
Chosen pressure (cm $\left.\mathrm{H}_{2} \mathrm{O}\right)$ & $8.7(2.5)$ & $8.2(2.1)$ \\
$\quad$ Successful & $8.8(2.4)$ & $8.1(2.1)$ \\
Undecided & $9.2(3.0)$ & $8.7(2.3)$ \\
Failed & $7.3(1.6)$ & (only 1 \\
& & subject) \\
Follow up data at 6 weeks: & & \\
Outcome & & \\
$\quad$ Successful & $39(64 \%)$ & $37(73 \%)$ \\
$\quad$ Undecided & $14(23 \%)$ & $13(25 \%)$ \\
$\quad$ Failed & $8(13 \%)$ & $1(2 \%)^{*}$ \\
Epworth Sleepiness Scale & & \\
$\quad$ Successful & $7.3(3.0)$ & $8.9(4.4)$ \\
$\quad$ Undecided & $12.7(4.9)$ & $15.8(4.6)$ \\
$\quad$ Failed & $13.0(6.3)$ & (only 1 \\
& & subject) \\
\hline
\end{tabular}

$>4 \% \mathrm{SaO}_{2}$ dips $/$ hour $=$ number of $>4 \%$ falls in percentage arterial oxygen saturation from the previous high value over the diagnostic night divided by the length of the study in hours; pulse rises/hour $=$ number of rises in pulse rate of $>7$ beats per minute over the diagnostic night, an index of sleep disturbance; $\mathrm{BMI}=$ obesity index, weight in kilograms divided by height in metres squared; Epworth Sleepiness Scale $(0=$ not sleepy at all, $24=$ maximum score, up to 9 regarded as within the normal range). One subject in the automatic group was not available for follow up.

$*$ p $<0.05$, manual versus automatic CPAP.

has remained untraceable, leaving 112 for the analysis of outcome.

No significant differences were seen between the two groups (table 1). Although the oxygen desaturation rate $\left(>4 \% \mathrm{SaO}_{2}\right.$ dips/hour) was non-significantly higher in the manual group, the Epworth Sleepiness Scale was very similar in the two groups. The CPAP pressures chosen were not significantly different between the two groups. The average pressure in the automatic group was about $0.5 \mathrm{~cm} \mathrm{H}_{2} \mathrm{O}$ lower which may be a reflection of the slightly lower severity of sleep apnoea in this group.

The outcomes were essentially similar with a slight trend in favour of the automatic titration. There were significantly fewer complete failures in the automatic titration group. Analysing just the subgroup who were successfully established on NCPAP at follow up, there were no significant differences in the mean (SD) scores on the Epworth sleepiness scale between the manual and automatic groups (manual 7.3 (3.0), automatic $8.9(4.4)$ ) indicating equal symptomatic benefit.

\section{Discussion}

These results show that the medium term outcome following automatic CPAP titration by the DeVilbiss Horizon system is as good as following manual titration and may be a little better. Since the final chosen NCPAP pressures were very similar and first night effects are unlikely to extend beyond six weeks, this suggests that automatic titration is likely to be as good as manual in the longer term as well. NCPAP use at one month has been shown to predict accurately subsequent use at three months. ${ }^{10}$
The slightly greater disease severity (as judged by the $>4 \% \mathrm{SaO}_{2}$ dips/hour) in the manual group may have biased outcome, but most data suggest that increasing severity improves NCPAP compliance rather than decreasing it, ${ }^{11}$ although not all groups have found this. ${ }^{12}$

It is not established how to select exactly the right NCPAP pressure during a titration night - for example, should the pressure chosen abolish all obstructive events, including inspiratory flow limitation, even at the expense of higher and thus more uncomfortable pressures? Following the institution of NCPAP patients can sleep without sleep disturbance even in the presence of inspiratory flow limitation, ${ }^{13}$ so automatic NCPAP machines that use this signal to control their pressure may be over treating. ${ }^{5}$ During NCPAP titration there are about $2 \mathrm{~cm}$ $\mathrm{H}_{2} \mathrm{O}$ hysteresis in the relationship between pressure and upper airway resistance depending on whether the pressure is being raised or reduced. ${ }^{14}$ Furthermore, the falling pressure requirements over the first few weeks of treatment ${ }^{15}$ and the possible variation in pressure required due to changes in nasal resistance, alcohol consumption, and sleep deprivation make obsessional titration on the first night superfluous. It is our experience that establishing patients successfully on NCPAP depends more on their training and support than on the very subtle adjustment of NCPAP pressure. The replacement of valuable technician time with an automatic alternative allows the technicians' expertise to be put to better use training and supporting patients. A recent small study looking at the effects of automatic versus fixed CPAP over a three week period at home (following a manual titration in the laboratory) did not find any differences in objective outcomes (such as the maintenance of wakefulness test) but found a small increase in compliance. ${ }^{6}$ This study was clearly looking at a different use for automatic CPAP machines but confirms that there appear to be no disadvantages in using them.

The results of this study show that replacing a skilled technician with an automatic NCPAP machine to establish the correct fixed pressure for a patient's subsequent use at home does not reduce the likelihood of the patient accepting NCPAP at six weeks. This has an important cost saving potential. The DeVilbiss Horizon automatic NCPAP machine appears capable of establishing sensible pressures, but at present this requires a manual review of the data on the following morning.

\footnotetext{
1 Montserrat JM, Alarcon A, Lloberes P, Ballester E, Fornas C, Rodriguez Roisin R. Adequacy of prescribing nasal continuous positive airway pressure therapy for the sleep continuous positive airway pressure therapy for the sleep apnoea/hypopnoea syndrome on the basis of night time respiratory recording variables. Thorax 1995;50:969-71.

2 Davies WL, Stradling JR. Auto-CPAP: comparison with constant pressure. $\mathcal{F}$ Sleep Res 1994;3(Suppl 1):57 (abstract).

3 Berthon Jones M. Feasibility of a self-setting CPAP machine. Sleep 1993;16:S120-1.

4 Miles LE, Buschek GD, McClintock DP, Miles SC, Narvio LR, Wang YX. Development and application of automatic nasal CPAP calibration procedures for use in the unsupervised home environment. Sleep 1993;16:S118-9. 5 Teschler H, Thompson AB, Henkel A, Berthon-Jones M,
} 
Konietzko N. Continuous positive airway pressure titration for obstructive sleep apnea syndrome by a selfsetting device. Am f Respir Crit Care Med 1995;151:A534.
Meurice JC, Marc I, Series F. Efficacy of auto-CPAP in the treatment of obstructive sleep apnea/hypopnea syndrome. Am 7 Respir Crit Care Med 1996;153:794-8.

7 Pepin JL, Nadjafizadeh H, Benkheira N, Levy P. A selfadjusted CPAP device for OSA using snoring detection and pressure changes: clinical validity. Am $\mathcal{f}$ Respir Crit Care Med 1995;151:A534.

8 Juhasz J, Schillen J, Urbigkeit A, Penzel T, Becker H, Peter $\mathrm{JH}$. Maschinenunterstutzte ermittlung des individuell
effectiven minimalen CPAP-Druckes mittels VitalogHMS-5000-Monitor. Pneumonologie 1995;49(Suppl 1): 199-204.

9 Johns MW. Daytime sleepiness, snoring, and obstructive sohns MW. Daytime sleepiness, snoring, and obstructive
sleep apnea. The Epworth sleepiness scale. Chest 1993;

103:30-6.
10 Kribbs NB, Pack AI, Kline LR, et al. Objective measurement of patterns of nasal CPAP use by patients with obstructive sleep apnea. Am Rev Respir Dis 1993;147:887-95.
11 Rolfe I, Olson LG, Saunders NA. Long-term acceptance of continuous positive airway pressure in obstructive sleep apnea. Am Rev Respir Dis 1991;144:1130-3.

12 Waldhorn RE, Herrick TW, Nguyen MC, O'Donnell AE, Sodero J, Potolicchio SJ. Long-term compliance with nasa continuous positive airway pressure therapy of obstructive sleep apnea. Chest 1990;97:33-8.

13 Montserrat JM, Ballester E, Olivi H, et al. Time-course of stepwise CPAP titration. Behavior of respiratory and neurological variables. Am f Respir Crit Care Med 1995; 152:1854-9.

14 Condos R, Norman RG, Krishnasamy I, Peduzzi N, Goldring RM, Rapaport DM. Flow limitation as a noninvasive assessment of residual upper-airway resistance during continuous positive airway pressure therapy of obstructive sleep apnea. Am f Respir Crit Care Med 1994; 150:475-80

15 Monton C, Montserrat JM, Parra O, Kimoff J, Cosio M A decrease in the level of CPAP required after prolonged treatment in patients with the obstructive sleep apnea syndrome. Arch Bronconeumol 1994;30:385-9. 